ARTICLE

\title{
The role of patient management in medical expertise development: Extending the contemporary theory
}

\author{
Alireza Monajemi MDa and Remy M.J.P. Rikers PhD \\ a Assistant Professor, Medical Humanities Research Group, Philosophy of Science Department, Institute for \\ Humanities and Cultural Studies, Tehran, Iran \\ b Professor, Department of Psychology, Erasmus University, Rotterdam, The Netherlands
}

\begin{abstract}
Medical expertise research has focused on why experienced physicians display remarkable performance in solving clinical problems and how this competency develops over many years of education and practice. The contemporary theory on the development of expertise in medicine proposes that students progress through different stages of knowledge restructuring (i.e., encapsulation and illness script) in which their knowledge is finely tuned towards practical situations. Although research in clinical case paradigm has provided us with a better understanding of the differences between students and doctors, it has almost exclusively focused on diagnostic performance, and patient management has received substantially less attention. This reflects an incomplete picture of a medical expert, as management is not only a crucial step in patient workup, but it is also highly connected to experience with patients. In their daily practice, doctors always diagnose and manage patients concurrently; hence, after a large number of patient encounters in practical settings, these two types of knowledge become integrated into one another. In order to incorporate management into contemporary theory, it is proposed that it should be considered as a part of the illness script structure. In the way towards expertise, knowledge about patient management becomes more prominent, and so an investigation of management knowledge could ultimately enable us to broaden our understanding of medical expertise.
\end{abstract}

\section{Keywords}

Medical Expertise, Encapsulation, Illness Script, Diagnosis, Patient Management

\section{Correspondence Address}

Alireza Monajemi, Institute for humanities and cultural studies, 64 ${ }^{\text {th }}$ Street, Kurdestan Expressway, Tehran, Iran, PO Box:14155-6419, Postal Code:14377-74681. E-mail: Monajemi@ihcs.ac.ir

Accepted for publication: 20 January 2011

\section{Introduction}

The research area that attempts to understand how doctors are able to deal with such diverse and complex problems is called medical expertise research. It has focused on why experienced physicians display such remarkable performance and how this competency develops over many years of education and practice [1-3]. This type of research can have significant impact on medical education as it guides medical educators on how to train medical students.

Although research on the development of medical expertise has a long tradition [1,3,4], medicine is obviously not the only domain that expertise research has been carried out on. One of the most dominant and influential domains of expertise research is chess, pioneered by the Dutch psychologist and chess master Adriaan de Groot. In his studies, he presented a chess position for a short period of time to chess players of different levels of expertise and asked them to think about the next move [5]. The strongest players (i.e. chess masters), unlike novices, were able to identify the best next move. Another task given to the participants was that they were asked to reconstruct pieces on the board after they had been removed from the board. This recall task was found to sharply discriminate between masters and non-masters, as non-masters failed to reproduce the position, while masters were able to put the pieces back on the right position.

The superiority of chess masters in reproducing the positions can be explained by their ability to perceive the pieces in clusters, and to recognize patterns of positions that they had seen before [6]. This superiority of experts in dealing with familiar patterns has been shown in a broad 
Table 1 Case description of Congestive Heart Failure(CHF)

A 70-year old female is admitted into the hospital because of increasing shortness of breath. History taking reveals that the patient has been very tired lately and tolerates her food badly. Sometimes she has chest pain, especially after dinner. She has admitted several times in C.C.U. The history of hypertension and hyperlipidemia is positive. Physical examination shows a pale and tired woman. She has an irregular, unequal pulse of $100 / \mathrm{min}$. The blood pressure is $110 / 70 \mathrm{mmHg}$ and Jugular Venous Pressure (JVP) is elevated. The patient has widespread peripheral edema and positive jugular venous pulsations. The heart is enlarged to all sides, and auscultation reveals a holosystolic murmur at the apex radiating towards the axilla. Lungs auscultation reveals rales at both sides at bases. The thoracic X-ray shows congestion of the lungs and an enlarged heart. Echocardiography shows an enlarged left atrium and ventricle. ECG reveals atrial fibrillation.

array of domains such as thermal-hydraulics [7], air traffic control [8], architecture [9], circuit techniques [10], sports $[11,12]$ and art [13]. Strongly influenced by the pioneering research in chess, in studies in these domains, participants of different expertise levels were also confronted with a task that could be evaluated (like the accuracy of a chess move) and was often followed by a recall task [14]. In other words, the paradigm that was introduced with chess studies was translated to many other areas of expertise research. In medicine, for instance, participants had to study a clinical case description (see Table 1), provide an accurate diagnosis, and eventually recall and explain the information in the case (i.e., free recall).

\section{Contemporary theory of medical expertise}

In particular, in these studies, doctors and medical students were asked to read a clinical case description for a few minutes followed by a diagnosis. Next they were asked to recall whatever they remembered from the case and to explain the signs and symptoms displayed in the case (i.e., pathophysiological explanation). This approach to study medical expertise is called the clinical case paradigm [1520]. Examples of pathophysiological protocols provided by a doctor and a medical student are presented in Table 2 .

As was expected, the results showed that experienced doctors always diagnosed the case more accurately than medical students. The novices' performance was also predictably below than that of the other groups. However the results from the students at an intermediate level were rather counterintuitive and unlike the findings in similar studies in other domains of expertise. They recalled more and explained the signs and symptoms more elaborately than both the doctors and novices. The question that arises here is that if doctors know more about everything in medicine than medical students, then why do they recall less than medical students? The analysis of the protocols uncovered the cause of this finding in medicine (see Table 2). It showed that doctors and students used different types of knowledge while solving clinical cases. That is, doctors use their clinical knowledge (i.e., the ways in which a disease manifests itself) to understand the problem, whereas, medical students apply their biomedical knowledge (i.e., the basic mechanisms and functioning of the human body and pathological process underlying the manifestation of diseases).

\section{Table 2 Pathophysiological explanation of the fourth-year medical students and the internist (expert doctor)}

\section{Pathophysiological explanation of the fourth-year medical students:}

As a result of increased pressure in pulmonary arteries due to the pressure and volume overload in left atrium and ventricle, the blood flows from left side of the heart and subsequently backs up in the pulmonary circulation. This leads to a congestion of lungs and extravagation of the fluid and causes shortness of breath in the patient.

\section{Pathophysiological explanation of the internist:}

Both the anemia and atrial fibrillation have worsened the forward and backward heart failure of the patient that leads to pulmonary edema.

In the protocol produced by a fourth-year medical student displayed in Table 2, he has focused on isolated signs and symptoms (such as edema or dyspnea) and seems to have attempted to understand the case by relating each of these to some pathophysiological mechanism (i.e., mechanisms of edema or mechanisms of pump failure). This is because students acquire extensive biomedical knowledge through courses in the basic sciences at the first stages of their medical training. Therefore, in order to make sense of the information provided in a clinical case, they will use their detailed, elaborate biomedical knowledge and thus they are more likely to remember the details better than doctors. As a result of this detailed processing as well as a lack of relevant knowledge, the fourth-year student will experience more difficulty in providing an accurate diagnosis. On the other hand, the doctor does not explicitly refer to the biomedical concepts anymore in the protocol (see Table 2). Examination the doctor's protocol shows that a few clinical concepts like forward failure or venous congestion were used to explain the case. These concepts are sufficient to understand all relevant signs and symptoms without the need to engage in a detailed biomedical mode as most students do. This is why the protocols of experts contain hardly any biomedical concepts but mainly clinical concepts. Schmidt and Boshuizen [21,22] have called these concepts "encap- 
sulated" because they summarize such biomedical knowledge under diagnostic labels (e.g., forward failure and pulmonary edema) which are simplified causal models that explain signs and symptoms. These encapsulated concepts develop as a result of extensive application of biomedical knowledge and especially through encounter with patient problems in medical diagnostic situations [3, 19-22].

However, knowledge encapsulation is not the last stage in the course towards expertise. Having encapsulated concepts such as forward failure is not enough to enable doctors to deal with real patients. Instead of using biomedical knowledge, the features that characterize the clinical presentation of a disease become the anchor points of experts' reasoning. An expert's knowledge is much richer than encapsulated knowledge, and it contains much more information about all different facets of diseases: about how diseases are acquired, how they manifest in patients, and which risk factors predispose them. When doctors would see a patient like the older woman described in Table 1, a diagnosis of Congestive Heart Failure (CHF) quickly pops into their mind as they recognize the pattern of the clinical presentation of the disease that has been formed as a result of having seen many similar patients.

\section{Table 3 The Illness script of Congestive Heart Failure (CHF)}

\section{Congestive Heart Failure(CHF)}

Faults: forward failure, backward failure, venous congestion, pulmonary edema, right-sided heart failure, left-sided heart failure

Consequences: dyspnea, chest pain, peripheral edema, raised JVP, indigestion, cardiomegaly

Enabling conditions: old age, previous coronary artery diseases, previous CCU admission, smoking, hypertension, hyperlipidemia

All the information about diseases that doctors have is organized in a structure called the illness script. It is an integrated knowledge structure consisting of at least three parts: faults, consequences, and enabling conditions [23]. Faults are pathophysiological malfunctions that constitute the biomedical core of the disease and are usually subsumed under a diagnostic label (e.g., forward failure, pulmonary edema). Consequences are about the clinical manifestations of a disease such as complaints, signs and symptoms (e.g. chest pain, dyspnea, fatigue). Finally, enabling conditions are the patient's background information that generally makes the occurrence of a certain disease more or less likely (e.g., age, sex, medical history, drug history, family history of diseases, occupation, and living environment [24-26]. For the CHF case, hypertension, hyperlipidemia and old age are enabling conditions. Table 3 shows the illness script of congestive heart failure.

Illness scripts and encapsulated knowledge are formed during the course of years of training and practice, hence, they differ strongly between students and doctors $[3,27,28]$. In the early stages of medical expertise development, biomedical knowledge plays an important role in constructing scripts for diseases. As students begin to practice with actual patients, their biomedical knowledge becomes encapsulated and will be reorganized into the illness scripts (i.e. fault section). In this phase, the newly formed illness scripts consist of signs, symptoms, and complaints (i.e., consequences) that are held together by a network of biomedical explanations.[25] With increasing expertise, the role of biomedical knowledge becomes less important; while simultaneously the role of enabling condition of diseases becomes more important. The integration of enabling conditions into illness scripts is a consequence of long period of clinical practice with real patients [3,25,27]. Enabling conditions give valuable information on how to quickly recognize diseases in daily practice. In the example mentioned above on congestive heart failure for instance, several risk factors like hypertension, hyperlipidemia, and previous cardiac problems in an old patient with increasing shortness of breath, are crucial to the doctor in picking CHF as the diagnosis, whereas in the absence of the above enabling conditions, in patient with an increasing shortness of breath, diagnostic hypotheses such asthma attack, pneumonia, pulmonary emboli, or COPD become more relevant. As a result of limited experience with actual patients in medical students, their knowledge about enabling conditions is not yet integrated in their still developing illness scripts. Consequently, they cannot benefit to the same extent as experts from the enabling conditions when diagnosing a case. In sum, the presented theory on the development of expertise in medicine proposes that students progress through different stages towards expertise. The encapsulated knowledge and illness script are two major processes of knowledge restructuring in which the medical students' knowledge is finely tuned towards practical situations to deal with real patients [3].

\section{Patient management and medical expertise theory}

Although research in the clinical case paradigm has provided us with a better understanding of the differences between the case representation in students and doctors, it has almost exclusively focused on diagnostic performance [15,18-20,22,29]. According to some authors [1,3,30-32], this is a limited perspective as patient management has received much less attention. This is in addition to the fact that patient workup consists of not only making a diagnosis, but also putting forward a management plan for the patient $[30,33]$. Doctors do much more than providing 
a diagnosis and a large part of their activity consist of figuring out management plans for patients [30]. Table 4 shows a Management plan for the case provided by one of the expert participants.

\section{Table 4 Management protocol of a patient with Congestive Heart Failure (CHF)}

\begin{tabular}{|ll|}
\hline 1.Semi sited position & 7.Digoxin \\
3. Low fat/low salt diet & 8.DC shock standby \\
$\begin{array}{l}\text { 4.Vital sign control } \\
\text { every } 30 \text { minutes }\end{array}$ & $\begin{array}{l}\text { 10.Angiotensin-converting } \\
\text { enzyme(ACE) inhibitors }\end{array}$ \\
$\begin{array}{l}\text { 5.Digitalization if } \\
\text { necessary }\end{array}$ & 11.Diuretics \\
$\begin{array}{l}\text { 6.Intermittent elastic } \\
\text { banding }\end{array}$ & $\begin{array}{l}\text { 12.Anticoagulation with heparin } \\
\text { and warfarin if necessary }\end{array}$
\end{tabular}

It is obvious that providing the correct diagnosis, without managing the immediate needs, is not sufficient and may lead to the patient's death. For instance, in the case above, because the patient is in pulmonary edema, it is imperative that a diuretic (e.g., Lasix) be immediately administered to resolve this urgent problem, or else the patient will suffocate. Studies have also highlighted the importance of management in the patient workup. For instance, it has been shown that medical doctors who consistently provide effective management (i.e. low rates of complication and death, and high rates of recovery and healing) are recognized as experts [4,34]. Other studies have indicated that extensive practice with patients appears to be linked to effective patient management [35,36]. Overall, it could be concluded that patient management is not only an essential component of medical practice, but also highly connected to expertise in medicine. As indicated above, the contemporary theory has treated the concepts of medical expertise and diagnostic focus as interchangeable, something that would reflect an incomplete picture of what it means to be a medical expert. It seems especially important to medical expertise research to have a good understanding of the nature of management knowledge and its organization in doctors' minds. This is because the improved understanding in this area is considered essential in broadening the definition of a medical expert [37].

Taking the management focus into consideration would raise some new questions that should be addressed. For instance, in what way does management knowledge differ between experienced and less experienced doctors or students? Would a management focus lead to a different evaluation of the findings in a clinical case by a doctor?
What is the relationship between the diagnostic and the management knowledge during the course of development towards expertise?

Although previous studies in clinical case paradigm have not focused on the management task, reviewing them may reveal some findings that can be considered as a starting point. Some studies that investigated the structure of illness script already revealed a trace of management knowledge. For example, Boshuizen, Schmidt, Custers, and Van de Wiel [38] asked medical students and family physicians to describe the clinical picture of some diseases (this is not similar to previous clinical case studies because there was no diagnostic situation). Interestingly, about $6.5 \%$ of the generated items predominantly referred to patient management. These items were neglected and were not taken into consideration because they were not assigned to any known component of the illness script. In the works by Hobus et al. [26] and Custers et al. [25], which used the same method as above, the results showed that participants with higher levels of expertise provided more information concerning patient management. That is expected, as experienced doctors obviously possess more knowledge about patient management and this knowledge is part of their clinical knowledge. If management knowledge is a part of the experts' knowledge, then why has it not appeared in most of the clinical case studies? In previous studies when participants were not explicitly asked to provide a diagnosis, management items did appear in the protocols. This is due to the fact that in their daily practice, doctors always diagnose and manage patients concurrently, and after a large number of patient encounters in practical settings, these two types of knowledge become integrated into one another [32,39].

\section{Table 5 The updated version of Congestive Heart Failure (CHF) illness script}

\section{Congestive Heart Failure(CHF)}

Faults: pulmonary edema, right-sided heart failure, leftsided heart failure.

Consequences: Dyspnea, chest pain, peripheral edema, raised JVP, indigestion, cardiomegaly

Enabling conditions: old age, coronary artery diseases, previous CCU admission, smoking, hypertension Management: Digoxin, Diuretics, DC shock, I/O control, ACE, Anticoagulation with heparin and warfarin if necessary, Digitalization if necessary

So it can be concluded that management is a neglected component of an illness script and should be incorporated into the illness script structure. An improved version of the illness script theory, therefore, contains information about the clinical presentation (i.e. consequences), the pathophysiological mechanisms (fault), risk factors (enabling condition), and finally the management plan 
(management component). The Table 5 shows this updated version of CHF illness script.

The way in which management becomes a part of the illness script is comparable to the described process of integration of enabling condition into the illness script $[3,25,27]$. That is, Mx knowledge is not a part of embryonic (newly formed) illness script of medical students, but as a result of extensive practice in patient management situations, it becomes integrated into the illness script structure and knowledge about patient management becomes more prominent. So it seems that the illness script of medical students is different from that of doctors with respect to their knowledge of patient management. A closer look at the course of medical education may reveal the origin of this difference. During the first years of the students' training (i.e., pre-clinical phase), there is a strong emphasis on providing correct diagnoses, which is often not accompanied by the same emphasis on developing a patient management plan $[32,40]$. The encounter of management tasks often starts considerably later (during the clinical phase) than that in diagnostic tasks. Consequently, less advanced students confronted with a management task, will most likely deal with it as if it were a diagnostic task because their scripts are tuned to solve diagnostic (Dx) problems.[32] More advanced students, on the other hand, might already have acquired some clinical experience in hospitals during their clinical rotations, and hence management has become a bit more concrete [32,39]. However, in contrast to experienced doctors these advanced students are still in the middle of the process of linking their diagnostic knowledge to their newly acquired management knowledge and they are not able to use their knowledge to solve management problems.

\section{Conclusion and implications for further research}

Although, as indicated, the patient management has received less attention in medical expertise research, the methods of clinical case studies could be adapted to study management knowledge acquisition and organization during the course towards expertise. A closer look at clinical case studies reveals that doctors and students, while processing case information, only had to work out what the case's diagnosis was. The ensuing recall and pathophysiological explanation therefore reflected how information was processed with a diagnostic focus. Changing this focus to a management focus could be obtained if participants are asked to read a case while thinking about patient management. Comparing these two case representations (i.e., diagnostic and management) may uncover the contribution of both to the development of medical expertise during education and practice. Further research may shed light on this neglected part of patient workup and may reveal that how management becomes a part of illness script and how it influences the way case information is processed. It can be concluded, therefore, that investigation into the doctors' and medical students' case representations in management could ultimately enable us to broaden our understanding of medical expertise.

\section{References}

[1] Norman, G. (2005) Research in clinical reasoning: past history and current trends. Medical Education, 39, 418-427.

[2] Schmidt, H.G., Norman, G. R., \& Boshuizen, H. P. A. (1990) A cognitive perspective on medical expertise: theory and implications. Academic Medicine, 65, 611-621.

[3] Schmidt, H.G., \& Rikers, R. M. J. P. (2007) How expertise develops in medicine: knowledge encapsulation and illness script formation. Medical Education, 41, 1133-1139.

[4] Ericsson, K. A. (2007) An expert-performance perspective of research on medical expertise: the study of clinical performance. Medical Education, 41, 1124-1130.

[5] De Groot, A. D. (1965) Thought and choice in chess. The Hague, The Netherlands: Mouton.

[6] De Groot, A.D., \& Gobet, F. (1996) Perception and Memory in Chess: Studies in the Heuristics of the Professional Eye. Van Gorcum, Assen.

[7] Vicente, K. J. (1992) Memory recall in a process control system: A measure of expertise and display effectiveness. Memory and cognition, 20, 33-57

[8] Morrow, D. G., Menard, W., Stine-Morrow, E. A. L., Teller, T., \& Byrant, D. (2001) The influence of expertise and task factors on age differences in pilot communication. Psychology \& Aging, 16, 31-46.

[9] Akin, O. (1980) Models of architectural knowledge. London: Pion.

[10] Egan, D. E., \& Schwartz, B. J. (1979) Chunking in recall of symbolic drawing. Memory \& Cognition, 7, 149-158.

[11] Allard, F., Graham, S., \& Parasalu, M. L. (1980) Perception in sport: Basketball. Journal of sport psychology, 2, 14-21.

[12] Eisenstaedt, M., \& Kareev, Y. (1975) Aspects of human problem solving: The use of internal representation. In Exploration in cognition (ed D.A. Norman., \& D. E. Rumelhart), pp. 308-346. San Francisco: Freeman.

[13] Krampe, R. T., \& Ericsson, K. A. (1996) Maintaining excellence: Deliberate practice and elite performance in young and older pianists. JEP: General, 125, 331-359.

[14] Vicente, K. J., \& Wang, J. H. (1998) An ecological theory of expertise effects in memory recall, Psychological Review, 105(1), 33-57.

[15] Boshuizen, H. P. A., \& Schmidt, H. G. (1992) On the role of biomedical knowledge in clinical reasoning by experts, intermediates and novices. Cognitive Science, 16, 153-184.

[16] Muzzin, L. J., Norman, G. R., Frighter, J. W., Tugwell, P., \& Guyatt, G. H. (1983) Expertise in recall of clinical protocols in two specialty areas. Proceedings of the $22^{\text {nd }}$ Conference on Research in Medical Education (pp. 122-127). Washington: American Association of medical Colleges.

[17] Norman, G. R., Brooks, L. R., \& Allen, S. W. (1989) Recall by experts and novices as a record of processing attention. Journal of Experimental Psychology: Learning, memory and Cognition, 1, 1166-1174. 
[18] Patel, V. L., \& Groen, G. J. (1986) Knowledge based solution strategies in medical reasoning. Cognitive Science, 10, 91-116.

[19] Rikers, R. M. J. P., Schmidt, H. G., \& Boshuizen, H. P.A. (2000) Knowledge encapsulation and the intermediate effect. Contemporary Educational Psychology, 25, 150-166.

[20] Schmidt, H. G., \& Boshuizen, H. P. A. (1993) On the origin of intermediate effects in clinical case recall. Memory and Cognition, 21(3), 338-351.

[21] Schmidt H. G., \& Boshuizen, H. P. A. (1993) On acquiring expertise in medicine. Educational Psychology Review, 5, 205221.

[22] Rikers, R. M. J. P., Schmidt, H. G., \& Boshuizen, H. P. A. (2002) On the constraints of encapsulated knowledge: Clinical case representation by medical experts and subexperts. Cognition and Instruction, 20 , 27-45.

[23] Feltovich, P. J., \& Barrows, H. S. (1984) Issues of generality in medical problem solving. In Tutorials in problem based learning (eds H. G. Schmidt., \& M. L. De Volder), pp. 128142. Assen, The Netherlands: Van Gorcum.

[24] Custers, E. J. F. M., Boshuizen, H. P. A., \& Schmidt, H. G. (1996) The influence of medical expertise, case typicality, and illness script component on case processing and disease probability estimates. Memory \& Cognition, 24, 384-399.

[25] Custers, E. J. F. M., Boshuizen, H. P. A., \& Schmidt, H. G. (1998) The role of illness scripts in the development of medical diagnostic expertise: results from an interview study. Cognition and Instruction, 16, 367-398.

[26] Hobus, P. P. M., Hosfstra, M. L., Boshuizen, H. P. A., \& Schmidt, H. G. (1988) De context van de klacht als diagnosticum [The context of the complaint as a diagnostic tool]. Huistarts en Wetenschap, 31, 261-267.

[27] Charlin, B., Boshuizen, H. P. A., Custers, E. J., \& Feltovich, P. J. (2007) Scripts and clinical reasoning. Medical Education, $41,1178-1184$.

[28] Charlin, B., Tardif, J., \& Boshuizen, H. P. A. (2000) Scripts and medical diagnostic knowledge: theory and applications for clinical reasoning instruction and research. Academic Medicine, 75, 182-190.

[29] Patel, V. L., Groen, G. J., \& Arocha, J. F. (1990) Medical expertise as a function of task difficulty. Memory and Cognition,
18, 394-406.

[30] Gruppen, L. D., \& Frohna, A. Z. (2002) Clinical reasoning. In International Handbook of Research in Medical Education (eds G. R. Norman., C.P.M. van der Vleuten., \& D. I. Newble), pp. 205-230. Dordrecht: Kluwer Academic Publishers.

[31] McGuire, C. H. (1985) Medical problem solving: a critique of the literature. Journal of Medical Education, 60, 587-595.

[32] Monajemi, A., Rikers, R. M. J. P., \& Schmidt, H. G. (2007) Clinical case processing: A diagnostic versus a management focus. Medical Education , 41, 1166-1172.

[33] Patel, V. L., Groen, G. J., \& Patel, Y. C. (1997) Cognitive aspects of clinical performance during patient workup: the role of medical expertise. Advances in health Science Education, 2, 95114.

[34] Ericsson, K. A. (2004) Deliberate practice and the acquisition and maintenance of expert performance in medicine and related domains. Academic Medicine, 79, 70-81.

[35] Norcini, J. J., Lipner, R. S., \& Kimball, H. R. (2002) Certifying examination performance and patient outcomes following acute myocardial infarction. Medical Education, 36, 853-859.

[36] Schurwirth, L., Gorter, S., van der Heijde, D., Rthans, J. J., Brauer, J., Houben, H., van der Linden, S., van der Vleuten, C., \& Scherpbier, A. (2005) The role of a computerized case based testing procedure in practice performance assessment. Advances in health Science Education, 10, 145-155.

[37] Albert, M., Hodges, B., \& Regehr, G. (2007) Research in medical education: balancing service and science. Advances in Health Sciences Education: Theory and Practice, 12 (1), 103115.

[38] Boshuizen, H. P. A., Schmidt, H. G., Custers, E. J. F. N., \& Van de Wiel, W. (1995) Knowledge development and restructuring in the domain of medicine: the role of theory and practice. Learning and instruction, 5, 269-289.

[39] Bissessur, S. W., Geijteman, E. C. T., Al-Dulaimy, M., Teunissen, P. W., Richir, M. C., Arnold, A. E. R., \& de Vries, T. P. G. M. (2009) Therapeutic reasoning: from hiatus to hypothetical model. Journal of Evaluation in Clinical Practice, 15, 985-989.

[40] Brater, D. C., \& Nierenberg, D. W. (1988) Medical students' education in clinical pharmacology and therapeutics. Annals of Internal Medicine, 108, 136-137. 